\title{
CÓMO DECIR MALAS NOTICIAS SIN FALTAR AL COMPROMISO CON LA HUMANIDAD DEL PACIENTE
}

\author{
Ybeth Luna-Solis 1,2,a
}

\begin{abstract}
RESUMEN
Comunicar malas noticias continúa siendo un reto en la comunicación en el campo asistencial y, pese a la existencia de metodologías que permiten humanizar una información de gran impacto en la vida de las personas, suele no ser enseñada ni utilizada en el quehacer diario, trayendo en consecuencia, desenlaces emocionales, algunas de ellos, más graves que la propia enfermedad. El arte de la comunicación, es un componente de la praxis médica, por ello la capacitación en los primeros años de la medicina sobre metodologías relacionadas a la comunicación de malas noticias incorporando la diversidad cultural, creencias y costumbres permitirá desarrollar habilidades en beneficio del paciente; aunque en determinados momentos es mejor no usar el lenguaje verbal, es importante recordar que el no verbal también puede transmitir el respeto que todo ser humano requiere ante la llegada de una mala noticia.
\end{abstract}

Palabras clave: Conductas relacionadas con la salud, Comunicación en salud, Humanismo, Pacientes (fuente: DeCS BIREME).

\section{HOW TO SAY BAD NEWS WITHOUT COMPROMISING THE PATIENT'S HUMANITY}

\begin{abstract}
Communicating bad news is still a communication challenge in the field of care and, despite the there being methodologies that allow us to humanize information that has a great impact on people's lives, it is usually neither taught nor used in daily life, thus bringing about emotional outcomes, that can be, at times, more serious than the disease itself. The art of communication is a component of medical praxis, so training in the early years of medicine on methodologies related to the communication of bad news incorporating cultural diversity, beliefs, and customs will develop skills for the benefit of the patient. Although at times it is better not to use verbal language, it is important to remember that nonverbal communication can also convey the respect that every human being requires before the arrival of bad news.
\end{abstract}

Keywords: Health-related behaviors, Health communication, Humanism, Patients (source: MeSH NLM).

\section{INTRODUCCIÓN}

La actividad asistencial suele estar compuesta de una fluida comunicación entre el paciente y el personal de salud, quienes deben informar de lo que se está realizando o ha de realizarse con el paciente. Sin embargo, ¿estamos preparados para comunicar una mala noticia sobre el estado de salud del doliente?

¿Cuántos de nosotros hemos recibido una mala noticia?, aquella información que no nos gusta, que con tan solo sentirla llegar $y$, por el impacto que tendrá en nuestras vidas, nos genera miedo, pero ¿cuántos de nosotros la hemos recibido de una manera tan directa, fría, demoledora y con falta de empatía? Aunque, se menciona que lo trascendente de una mala noticia es producto de nuestros propios temores, también es conocido que es vital la forma en que el interlocutor la dice y propicia el encuentro para mencionarla.

En nuestra experiencia, una mala noticia logra convertirse en todo lo existente, no hay condición que sea mayor ni responsabilidad que te aleje de ella. Te envuelve la tristeza, el miedo y, los pensamientos, incluso de muerte, se apoderan de quién la habría vivenciado.

Para dar malas noticias se requiere de una instrucción específica, habilidades de comunicación que, desafortunadamente, no forman parte de la enseñanza académica, por lo que, el médico

\footnotetext{
Instituto Nacional de Salud Mental “Honorio Delgado - Hideyo Noguchi”. Lima, Perú.

Facultad de Medicina, Universidad Peruana Cayetano Heredia. Lima, Perú.

Médico psiquiatra, maestra en Gerencia de Servicios de Salud.

Recibido: 10/09/2018 Aprobado: 27/02/2019 En línea: 11/03/2019
}

Citar como: Luna-Solis Y. Cómo decir malas noticias sin faltar al compromiso con la humanidad del paciente. Perú. Rev Peru Med Exp Salud Publica. 2019;36(1):123-7. doi:10.17843/rpmesp.2019.361.3921. 
desarrolla estrategias en función a su experiencia y muchas veces siguiendo sólo el principio ético de beneficencia, por el cual retiene u otorga información que considera relevante y protectora para el paciente ${ }^{(1)}$.

\section{¿QUÉ SON MALAS NOTICIAS?}

Una mala noticia es una información que logra frustrar o condicionar de manera negativa las expectativas de la persona que la recibe ${ }^{(1)}$. También se define como aquella información que genera una perniciosa transformación en la vida del paciente (2). Pueden considerarse como una mala noticia, el grave progreso de una patología, el diagnóstico de una enfermedad mortal no tratable o de larga evolución o la defunción de un familiar, por lo que es importante considerar que sin el conocimiento necesario para comunicarla puede llegar a ser nociva tanto para el paciente como para el propio médico ${ }^{(3)}$ y logra dificultar la comprensión de la intervención y el compromiso del paciente en su tratamiento ${ }^{(4)}$.

Comunicar malas noticias logra producir estrés en el médico ${ }^{(2)}$ o también éste puede no llegar a percibirla como una intervención de gran impacto en la vida del paciente ${ }^{(1)}$. En el primer caso, para evitarse problemas puede ocultar las malas noticias ${ }^{(2)}$; mientras que, en el segundo, brindar información exhaustiva, no requerida por el paciente, genera nuevos conflictos en la calidad de vida del informado ${ }^{(1)}$.

En algunas especialidades médicas, la comunicación de malas noticias es más compleja, por la existencia de factores como el temor del médico por experiencias previas, el estado clínico del paciente y las reacciones emocionales del paciente y sus familiares, las cuales terminan generando serias dificultades para la comunicación de la noticia o acudiendo a perífrasis para evitar terminologías que desesperancen al paciente ${ }^{(5)}$. Sin embargo, el interés del paciente por conocer la información completa es cada vez mayor, desvinculándose de la dinámica paternalista hasta asumir un rol más activo sobre su enfermedad, para lo cual se va requerir del médico valores relacionados a la honestidad, compasión y esperanza, pero principalmente, el compromiso sincero para la entrega de malas noticias ${ }^{(6)}$.

En la actualidad, la información al paciente se debe brindar siguiendo el principio ético de autonomía, tangible a través del consentimiento informado, que direcciona la aplicación de valores como la honestidad y la búsqueda del empoderamiento del paciente para la toma correcta de decisiones ${ }^{(1,7)} \mathrm{y}$, el principio de justicia, que establece como responsabilidad del médico informar todo lo referente al derecho e interés del paciente ${ }^{(1)}$. Sin embargo, en la cotidianidad se termina favoreciendo el principio de no maleficencia con menoscabo de los otros principios ${ }^{(8)}$.

\section{CONTEXTO RELACIONADO A LA COMUNICACIÓN DE MALAS NOTICIAS}

En diversos estudios se ha evaluado la capacidad del médico para comunicar malas noticias ${ }^{(2,7)}$. Sin embargo, a pesar de los esfuerzos de mejorar las competencias en la comunicación, aún no se perciben lo suficientemente capacitados para lograrlo (2).

En el estudio de Outram et al., en dieciséis profesionales en salud mental, pese a reconocer la importancia de brindar toda la información al paciente y su relación con la adherencia al tratamiento, algunos evitaban darlas, expresando diversas razones, principalmente relacionadas al temor de cómo la información sería recibida por los pacientes y sus familiares y al estigma que se asocia con un determinado diagnóstico. Concluyeron que un entrenamiento sobre cómo comunicar malas noticias permitiria al clínico reconocer sus propios sentimientos y adquirir habilidades en la comunicación ${ }^{(5)}$.

En nuestro medio, esta situación es más desmoralizadora, en el estudio de Bardales et al., hallaron que la mayoría de 317 profesionales de salud encuestados de un hospital de nivel resolutivo III-1, si bien conocían sobre lo que es comunicar malas noticias y lo difícil de su proceso, el $72 \%$ mencionaron no haber recibido capacitación para hacerlo ${ }^{(7)}$. En el estudio de Guillén-López y OlascoagaMesía, hallaron que los médicos residentes de un hospital de alto nivel resolutivo, no recibieron capacitación para comunicar malas noticias pese a que la dinámica asistencial exigía hacerlo, siendo los médicos en proceso de formación los que mayoritariamente las proporcionaban. Asimismo, fueron las falsas expectativas de los pacientes y la carencia de espacios adecuados, las principales limitaciones para comunicar malas noticias. Las emociones como frustración y temor se manifestaron como sentir del médico al comunicarlas ${ }^{(3)}$.

En el mismo estudio, Guillén-López y Olascoaga-Mesía consideraron que la capacitación en etapas tempranas de la universidad, reforzadas en el campo clínico, con espacios idóneos y con apoyo de médicos con mayor experiencia deben ser los pilares del acercamiento al paciente para comunicar malas noticias ${ }^{(3)}$. Del mismo modo, Monge y Sotomayor, hallaron en un hospital general, en pacientes $(n=42)$, familiares $(n=30)$ y personal médico de cuidado oncológico $(n=31)$, que el deseo del paciente fue ser comunicado sobre su diagnóstico; mientras que, los familiares y médicos prefirieron la no comunicación de esa información al paciente y así evitarle angustia y un posterior deterioro de su condición médica ${ }^{(8)}$.

Sobre los factores que contribuyen en la comunicación de malas noticias, Aranda et al., los agruparon, en aquellas relacionadas con el médico (tipo de comunicación 
verbal, no verbal, saber sobre el paciente y su patología, experiencia obtenida, estudios previos, entre otros), con el paciente (tiempo para comprender la información, estado de la enfermedad, apoyo familiar, tipo de personalidad, entre otros) y con el contexto en las que se comunican (necesidad de un espacio privado). Asimismo, señalaron que comunicar malas noticias pudiera generar el fortalecimiento de la relación médico-paciente o su quiebre, si éstas no son informadas de manera correcta ${ }^{(9)}$.

Para Guerra-Tapia y González-Guerra, las condiciones previas a una mala noticia permiten obtener una comunicación personalizada y direccionada a la humanidad del paciente ${ }^{(1)}$ :

1. Conocimiento preciso sobre el estado de salud del paciente.

2. Conocimiento sobre las condiciones personales y contextuales del paciente.

3. Planteamiento de estrategias teniendo apoyo de los familiares del paciente.

4. Relación médico-paciente y confidencialidad garantizadas en la dinámica de la intervención.

\section{ABORDAJE METODOLÓGICO SOBRE COMUNICAR MALAS NOTICIAS}

En la actualidad existen diversos modelos de intervención para dar una mala noticia, siendo las principales las que se describen a continuación.

Para cumplir con los objetivos fundamentales de conocer al paciente y las esperanzas presentes en él, brindar información clara, disminuir el impacto emocional y poder contar con la participación del paciente, Baile et al., desarrollaron un protocolo compuesto de seis pasos llamado SPIKES, por las iniciales en inglés, principalmente para pacientes oncológicos, con posibilidad de ser adaptado a diversos campos clínicos ${ }^{(10)}$ :

- Paso 1 (S): preparar la entrevista mediante una elaboración mental de cómo iniciar la comunicación e imaginar las reacciones del paciente. Desarrollar algunas actividades como ubicar un espacio para la privacidad de la comunicación, inclusión de familiares, comodidad y gestión para proporcionar el tiempo necesario y evitar las interrupciones; pero principalmente, lograr el contacto emocional con el paciente mediante el contacto físico, sosteniendo la mano o tocando el brazo y, visual, que permitan la disminución del sentimiento de frustración o responsabilidad del médico por ser el interlocutor de las mismas.

- Paso $2(\mathrm{P})$ : realizar preguntas previas para evaluar y conocer la percepción e información que tiene el paciente sobre su enfermedad, su nivel de comprensión y expectativas.
- Paso 3 (I): conocer la necesidad de información que requiere el paciente, sea ésta mucha o poca, que permita al médico la entrega de las malas noticias; por lo que, preguntas sobre qué, cuánto y cómo saber, son parte de las estrategias de este paso.

- Paso $4(\mathrm{~K})$ : insinuar la existencia de las malas noticias puede lograr alertar al paciente y disminuir su impacto en él. Son necesarios el uso de una comunicación no compleja, pausada y evitando frases desalentadoras.

- Paso 5 (E): ante las distintas reacciones emocionales del paciente, el médico debe mostrar una posición empática, lográndose, mediante la observación, la identificación de la emoción del paciente y la razón de dicha emoción.

- Paso 6 (S): contar con un plan de intervención permitiría reducir la incertidumbre de lo que ha de acontecer, permitiendo al paciente sentirse menos ansioso; sobre todo sí se le incluye como responsable de su tratamiento y se le permite la expresión de sus emociones y expectativas en relación a su enfermedad.

Si observamos detenidamente el protocolo de Baile et al. ${ }^{(10)}$, veremos que el proceso de la planificación, y la visualización de lo que ha de acontecer, es un paso esencial para comunicar malas noticias y lograr una menor afectación emocional de quién las comunica.

Para comunicar malas noticias, Bennett y Alison, (UK, 1996), plantearon el siguiente enfoque principalmente para pacientes con cáncer ${ }^{(11)}$ :

- Preparación previa de la comunicación, mediante consultas iniciales en la que se anticipen la presencia de malas noticias con expresiones de preocupación y expectativa, evitando en todo momento falsas esperanzas.

- Iniciar la comunicación teniendo en cuenta el lugar adecuado, el tiempo necesario y la documentación para explicar con claridad lo que está aconteciendo.

- Es necesario preguntar al paciente qué conoce de su enfermedad, cómo interpreta lo que le está sucediendo, sí desea saber más y, en caso de no desearlo, respetar su decisión, garantizando estar siempre en comunicación sí así lo desea.

- Explicar pausadamente lo que está ocurriendo, teniendo en cuenta la comprensión de la información por parte del paciente, repetirla si es necesario y explicar de la manera más real posible en qué consiste la enfermedad.

- La expresión emocional del paciente puede ser muy variada, desde manifestaciones de llanto e ira hasta el más profundo silencio, por lo que el médico debe manejar estas expresiones de forma empática.

- Evidenciar las preocupaciones que envuelven al paciente, por lo que responder garantizando el máximo de esfuerzo para resolverlos e ir planteando intervenciones convenientes son importantes. 
- El pronóstico de la enfermedad es parte de lo que se desea saber; sin embargo, dar fechas exactas sería un error. La mención de lo impreciso del tiempo y la diferencia entre lo que significa tratamiento y cura, mencionándolos con sensibilidad y comprensión son partes de las estrategias.

- Antes de finalizar la entrevista, el médico debe mencionar el apoyo continuo y la apertura a una nueva entrevista, sí así lo desea el paciente. La anotación correcta en la historia clínica o redacción de notas para otro personal asistencial es parte de ésta comunicación. Finalmente, se debe brindar unos minutos para recuperar la condición emocional del médico y proceder con la atención del siguiente paciente.

Hasta este punto, la única diferencia que existe entre el protocolo SPIKES y el realizado por Bennett y Alison, según Herrera et al., es que el primero, plantea una preparación mental del médico antes de comunicar una mala noticia; mientras que, la información comunicada con empatía, claridad, cuidando su comprensión y, la necesidad del apoyo emocional de los familiares, son pilares de ambas intervenciones ${ }^{(12)}$.

Aunque estas intervenciones son principalmente para comunicar malas noticias en el campo oncológico ${ }^{(10,11)}$, suelen ser utilizadas en todas las demás especialidades, dónde las malas noticias, son parte del proceso habitual de la actividad médica (11).

Para Guerra-Tapia y González-Guerra, los siguientes son pasos necesarios para brindar una mala noticia en cualquier campo asistencial ${ }^{(1)}$ :

- El análisis del entorno, mediante la obtención de un ambiente tranquilo, cómodo y cercano al paciente. Contando con el tiempo necesario.

- Conocer de dónde se parte, sabiendo qué conoce, qué desea conocer, aceptación de los silencios y negativas del paciente, con ofrecimiento de otras consultas médicas de requerirlo.

- Compartir la información, observando el lenguaje no verbal mediante la mirada, gestos, tono de voz, seguridad, amabilidad, comprensión; así como, el lenguaje verbal con información objetiva y concreta, pero a la vez enfatizando el entendimiento del mensaje y la escucha activa.

- Empatizando con el paciente sin llegar a la angustia por parte del médico.

- Ofrecimiento de cuidados y seguimientos médicos para el manejo de la incertidumbre.

Por otro lado, pese a la presencia de protocolos en comunicar malas noticias y los grandes esfuerzos de los médicos, nada asegura la forma en que los pacientes tomaran la información vertida, existiendo también el componente individual como un parámetro más de la comunicación ${ }^{(13)} \mathrm{e}$, incluso no es posible concluir que los entrenamientos sean totalmente beneficiosos para la adquisión de habilidades en la comunicación (2). Al respecto, en el estudio de Coutinho y Ramessur, sólo el $21 \%$ de 260 de estudiantes del quinto y sexto año de medicina de una universidad pública culminaron con un entrenamiento sobre cómo comunicar malas noticias. Los estudiantes mencionaron la necesidad de contenidos teóricos y prácticos. Además, señalaron que, el paso cuatro $(\mathrm{K})$, sobre la entrega de información para el paciente y el paso cinco $(E)$, relacionado a las emociones y empatía, del protocolo SPIKES fueron considerados los de mayor dificultad para ser aplicados en un contexto clínico ${ }^{(14)}$.

Es importante mencionar, que la comunicación de malas noticias requiere de metodologías educativas que garanticen el aprendizaje del estudiante, contar con protocolos adaptados a nuestra realidad y un sistema de valores en la que se sostenga una comunicación con humanidad. En ese sentido, ninguno de los protocolos mencionados consideran contextos con limitaciones de recursos como los nuestros y cómo deliberar en medio de esas dificultades.

\section{CONCLUSIONES}

Existen protocolos para comunicar malas noticias. Sin embargo, una condición común entre los profesionales de salud y principalmente entre los médicos, pese a la necesidad diaria de comunicarlas, es la escasez de capacitación en esta temática $y$, asociado a la falta de apoyo de médicos con mayor experiencia para informarlas, terminan contribuyendo a la carencia de cuidado ante una situación especial como es el comunicar malas noticias, trayendo en consecuencia, el quiebre en la calidad de atención del paciente y el cuidado emocional del propio médico responsable en hacerlo.

Por todo lo mencionado, es necesario mayor preparación para comunicar malas noticias, siendo importante la capacitación desde los primeros años de estudios de medicina, con temas sobre metodologías relacionadas a comunicar malas noticias, las cuáles permitirán desarrollar habilidades en base a intervenciones organizadas y estandarizadas. Asimismo, siendo Perú un país con diversidad cultural, creencias y costumbres, es necesario promover la adaptación o elaboración de metodologías dentro de un contexto latinoamericano. $Y$, si en medio de la comunicación nos quedamos sin saber qué hacer, el mantener silencio acompañado de una capacidad empática nos pueden permitir seguir conectados con el sistema emocional del paciente, dado que la comunicación, principalmente no verbal, puede brindar, aún en silencio, el respeto que todo ser humano requiere ante la llegada de una mala noticia.

Fuentes de financiamiento: Autofinanciado.

Conflictos de interés: La autora declara no tener conflictos de intereses en la publicación del artículo. 


\section{REFERENCIAS BIBLIOGRÁFICAS}

1. Guerra-Tapia A, González-Guerra E. Transmisión de malas noticias en la consulta. Actas Dermosifiliogr. 2013; 104 (1):1-3. https://doi.org/10.1016/j. ad.2012.09.002

2. Alelwani SM, Ahmed YA. Medical training for communication of bad news: A literatura review. J Edu Health Promot. 2014; 3:51. doi: 10.4103/22779531.134737.

3. Guillén-López OB, Olascoaga-Mesía AC. Habilidad de los médicos residentes para comunicar malas noticias. Rev Soc Peru Med Interna. 2017; 30 (2): 78-81.

4. Herrera A, Ríos M, Manríquez JM, Rojas G. Entrega de malas noticias en la Práctica clínica. Rev Med Chile. 2014; 142: 13061315. http://dx.doi.org/10.4067/S003498872014001000011.

5. Outram S, Harris G, Kelly B, Cohen M, Sandhu H, Vamos M, et al. Communicating a schizophrenia diagnosis to patients and families: A qualitative study of mental health clinicians. Psychiatric Services. 2014; 65 (4): 551-554. doi: 10.1176/appi.ps.201300202.
6. Spafford MM, Schryer CF, Creutz S. Balancing patient care and student education: Learning to deliver bad news in an optometry teaching clinic. Adv Health Sci Educ Theory Pract. 2009; 14 (2): 233250. doi: 10.1007/s10459-008-9107-5.

7. Bardales AF, Querevalú DL, MaqueraAfaray J. Comunicación de malas noticias: experiencia en un hospital del Perú. Rev Peru Med Exp Salud Pública. 2014; 31 (3): 603-4.

8. Monge E, Sotomayor R. Attitudes towards delivering bad news in Peru. Lancet. 2004; 363 (9420): 1556. https://doi. org/10.1016/S0140-6736(04)16167-9.

9. Aranda CI, Cedillo R, Del Campo MG, Omelas RO, Góngora J. Factores que influyen y dificultan la comunicación de malas noticias en el personal de salud. Acta Universitaria [Internet]. 2014 [Citado 09 de agosto de 2018]; 24 (5). Disponible en: https://www.redalyc.org/articulo. oa? $\mathrm{id}=41632450003$.

10. Baile WF, Bukman R, Lenzi R, Glober G, Beale EA, Kudelka AP. SPIKES-A SixStep Protocol for Delivering Bad News:
Application to the Patient with Cancer. The Oncologist. 2000; 5: 302-311. doi: 10.1634/theoncologist.5-4-302.

11. Bennett $M$, Alison D. Discussing the diagnosis and prognosis with cancer patients. Postgrad MedJ. 1996; 72(843):25-29.

12. Herrera A, Ríos M, Manríquez JM, Rojas G. Entrega de malas noticia en la práctica clínica. Rev Med Chile 2014; 142: 13061315. http://dx.doi.org/10.4067/S003498872014001000011.

13. Klyce W. On Breaking Bad News. JAMA. 2018; 320 (2): 135-136. doi:10.1001/ jama.2018.8544.

14. Coutinho F, Ramessur A. An Overview of Teaching Communication of Bad News in Medical School: Should a Lecture be Adequate to Address the Topic?. Acta Med Port. 2016; 29 (12): 826-831. doi: 10.20344/amp.7909.

Correspondencia: Ybeth Luna Solis

Dirección: Jr. Tamarugal 209 Urb. Tarapacá - Callao, Perú.

Correo electrónico: ybeth.luna.s@upch.pe

\section{Suscríbete a nuestro canal de Youltube y disfruta la mejor y más completa información de investigación científica} www.youtube.com/user/RPMESP 